\title{
ON RELATIVE CLASS NUMBERS OF CERTAIN QUADRATIC EXTENSIONS ${ }^{1}$
}

\author{
BY JIH-MIN SHYR
}

Communicated by Dock Rim, November 11, 1974

1. Introduction. As a generalization of the well-known class number formula of an algebraic number field, we obtain in [3] a formula for the class number of an arbitrary algebraic torus defined over the rational number field Q. With this generalized class number formula, the relative class number of two isogenous tori can be expressed in terms of their Tamagawa numbers and certain indices of maps induced naturally by an isogeny between them. To be specific, let $\lambda: T \rightarrow T^{\prime}$ be an isogeny of tori defined over $\mathbf{Q}$. The isogeny induces naturally the following maps (cf. [3]):

$$
\left.\lambda_{v}: T_{v} \rightarrow T_{v}^{\prime}, \quad \lambda_{v}^{c}: T_{v}^{c} \rightarrow T_{v}^{\prime c}, \quad \lambda_{\mathrm{Q}}^{\infty}: T_{\mathrm{Q}}^{\infty} \rightarrow T_{\mathrm{Q}}^{\prime \infty}, \hat{\lambda}\right)_{\mathrm{Q}}:\left(\hat{T}^{\prime}\right)_{\mathrm{Q}} \rightarrow(\hat{T})_{\mathrm{Q}}
$$

For a homomorphism $\alpha: G \rightarrow G^{\prime}$ of commutative groups with finite kernel and cokernel, we define the $q$-symbol of $\alpha$ by $q(\alpha)=[\operatorname{Cok} \alpha] /[\operatorname{Ker} \alpha]$. Then, the $q$-symbols of the above maps are finite, and $q\left(\lambda_{v}^{c}\right)=1$ for almost all $v$ (cf. [3]). The relative class number $h_{T} / h_{T^{\prime}}$ of $T, T^{\prime}$ over $\mathbf{Q}$ can now be expressed as (cf. [3]):

$$
\frac{h_{T}}{h_{T^{\prime}}}=\frac{\tau_{T}}{\tau_{T^{\prime}}} \cdot \frac{q\left(\lambda_{\infty}\right)}{\left.q\left(\lambda_{\mathrm{Q}}^{\infty}\right) q(\hat{\lambda})_{\mathrm{Q}}\right)} \cdot \prod_{v \neq \infty} q\left(\lambda_{v}^{c}\right) .
$$

In this paper, we apply (1) to the study of relative class numbers of certain quadratic extensions of algebraic number fields.

2. Relative class numbers. Let $k / Q$ be a finite extension, and $K / k$ be a Galois extension of finite degree $n$. Denote by $N$ the norm map $R_{K / k}\left(\mathrm{G}_{m}\right) \rightarrow \mathrm{G}_{m}$, where $R_{K / k}$ is the Weil functor of restricting the field of definition (cf. [4]), and $\mathbf{G}_{m}$ is the multiplicative group of the universal domain. We have an exact sequence $(N) 0 \rightarrow \operatorname{Ker} N \stackrel{i}{\rightarrow} R_{K / k}\left(\mathbf{G}_{m}\right) \stackrel{N}{\rightarrow} \mathbf{G}_{m} \rightarrow 0$ of

AMS (MOS) subject classifications (1970). Primary $12 \mathrm{A50}, 20 \mathrm{G} 30$.

${ }^{1}$ This paper is based on a part of the author's Ph.D. thesis written at Johns Hopkins University. For the unexplained notions, see [3]. 
tori defined over $k$, where $i$ is the canonical inclusion. We attach to $(N)$ an isogeny $\lambda^{\prime}: R_{K / k}\left(\mathbf{G}_{m}\right) \rightarrow \operatorname{Ker} N \times \mathbf{G}_{m}$ defined by $\lambda^{\prime}(x)=\left(x^{n} N(x)^{-1}, N(x)\right)$. Applying the Weil functor $R_{k / \mathrm{Q}}$ to $\lambda^{\prime}$, we have an isogeny $\lambda: T \rightarrow T^{\prime} \times T^{\prime \prime}$ defined over $\mathbf{Q}$, where $\lambda, T, T^{\prime}, T^{\prime \prime}$ denote $R_{k / \mathrm{Q}}\left(\lambda^{\prime}\right), R_{K / \mathrm{Q}}\left(\mathbf{G}_{m}\right), R_{k / \mathrm{Q}}(\operatorname{Ker} N)$, $R_{k / \mathrm{Q}}\left(\mathrm{G}_{m}\right)$ respectively. From (1), we obtain

THEOREM 1. Let $K, k, T^{\prime}, \lambda$ be as above. Then we have

$$
\frac{h_{K}}{h_{k}}=\frac{h_{T^{\prime}}}{\tau_{T^{\prime}}} \cdot \frac{q\left(\lambda_{\infty}\right)}{\left.q\left(\lambda_{\mathrm{Q}}^{\infty}\right) q(\hat{\lambda})_{\mathrm{Q}}\right)} \cdot \prod_{v \neq \infty} q\left(\lambda_{v}^{c}\right) .
$$

3. Relative quadratic extensions. If $K$ is a quadratic extension of $k$, we can interpret $h_{T}$ in the following way. Consider $K$ together with the norm $N_{K / k}$ as a binary quadratic space over $k$. Then $\operatorname{Ker} N$ is nothing but the special orthogonal group of the quadratic form $N_{K / k}$. By fixing a basis, we identify $K$ with $k^{2}$. A finitely generated $O_{k}$-submodule of $K$ of rank 2 is called a lattice in $K$, where $O_{k}$ is the ring of algebraic integers in $k$. In particular, $L=O_{k}^{2}$ (relative to the fixed basis) is a lattice, called the standard lattice. The adele group $(\operatorname{Ker} N)_{\mathrm{A}}$ and the group $(\operatorname{Ker} N)_{k}$ both act on the set of lattices in $K$ (cf. [2]). The orbit of $L$ under $(\operatorname{Ker} N)_{k}$ is called the class of $L$. The orbit of $L$ under $(\operatorname{Ker} N)_{\mathrm{A}}$ is called the genus of $L$. We see immediately that the number $h_{T^{\prime}}$ is the number of classes in the genus of $L$. Thus, in this case Theorem 1 establishes a relation among the class numbers of $K, k$, and the quadratic form belonging to $K / k$. In the following examples, we shall apply Theorem 1 to some interesting cases.

EXAMPLE 1 (Quadratic fields). Let $k=\mathbf{Q}$ and $K=\mathbf{Q}(\sqrt{m})$, where $m$ is a square-free integer. Computations using results in class field theory show that $\left.\left.\left.\tau_{T^{\prime}}=2, q(\hat{\lambda})_{Q}\right)=1, q \lambda_{\infty}\right)=1, q \lambda_{Q}^{\infty}\right)=2$ if $m<0$, or $m>0$ and $N_{K / \mathrm{Q}}(\epsilon)=-1$ for some $\epsilon \in O_{K}^{\times}, q\left(\lambda_{\mathrm{Q}}^{\infty}\right)=4$ if $m>0$, and $N_{K / \mathrm{Q}}(\epsilon)=1$ for some $\epsilon \in O_{K}^{\times}$, and $\Pi_{v \neq \infty} q\left(\lambda_{v}^{c}\right)=2^{t+1}$, where $t$ is the number of distinct prime factors of the discriminant $d_{K}$ of $K$. Hence, Theorem 1 is simplified to

$$
\frac{h_{K}}{h_{T^{\prime}}}= \begin{cases}2^{t-1} & \text { if } m<0 \text { or } m>0 \text { and } N_{K / Q}(\epsilon)=-1, \\ 2^{t-2} & \text { if } m>0 \text { and } N_{K / Q}(\epsilon)=1 .\end{cases}
$$

This is equivalent to the following well-known result due to Gauss: there are $2^{t-1}$ genera of primitive integral binary quadratic forms with discriminant $d_{K}$ (which are positive-definite if $d_{K}<0$ ). 
ExAmple 2 (Prime cyclotomic fields). Let $K$ be the $l$ th cyclotomic field over $\mathbf{Q}$, and $k$ the maximal totally real subfield of $K, l=2 m+1$ being an odd prime number. We prove in [3] that $q\left((\hat{\lambda})_{\mathbf{Q}}\right)=1, q\left(\lambda_{\infty}\right)=1, q\left(\lambda_{\mathbf{Q}}^{\infty}\right)=2^{m}$, $\Pi_{v \neq \infty} q\left(\lambda_{v}^{c}\right)=2^{m+1}$, and $\tau_{T^{\prime}}=2$. Therefore, Theorem 1 in this case gives $h_{K} / h_{k}=h_{T}$. Thus, we have an interpretation for the first factor in the wellknown factorization of $h_{K}$ due to Kummer. Moreover, we obtain the following:

THEOREM 2. Let $g$ be a primitive root modulo $l$. For any positive integer $s$, let $g_{s}$ denote the smallest positive residue of $g^{s}$ modulo $l$. Then there are

$$
\left(1 /(2 l)^{m-1}\right) \cdot\left|\operatorname{det}\left(g_{m+i+j}-g_{i+j}\right)_{0 \leqslant i, j \leqslant m-1}\right|
$$

classes in the genus of the standard lattice in $K$ with respect to the binary quadratic form $N_{K / k}$.

EXAMPLE 3 (Totally imaginary quadratic extension of a totally real field). Let $k$ be a totally real number field, and $\delta$ a totally negative number in $k$. Then $K=k(\sqrt{\delta})$ is a totally imaginary quadratic extension of $k$. Hecke conjectured that the relative class number $h_{K} / h_{k}$ should be expressible in terms of elementary arithmetic functions of certain types (cf. [1]). We hope that the relation described in Theorem 1 may throw a new light on Hecke's conjecture.

\section{REFERENCES}

1. E. Hecke, Bestimmung der Klassenzahl einer neuen Reihe von algebraischen Zahlköpern, Göttingen Nachr. 1921, 1-23.

2. T. Ono,.Sur une propriété arithmétique des groupes algébriques commutatifs, Bull. Soc. Math. France 85 (1957), 307-323. MR 20 \#880.

3. J. Shyr, Class number formulas of algebraic tori with applications to relative class numbers of certain relative quadratic extensions of algebraic number fields, Ph. D. Thesis, Johns Hopkins University, 1974.

4. A. Weil, Adeles and algebraic groups, Lecture notes, Princeton.

DEPARTMENT OF MATHEMATICS, JOHNS HOPKINS UNIVERSITY, BALTIMORE, MARYLAND 21218 